Overall recurrence rates at two years were $12.3 \%$ lobectomy, $11.8 \%$ sub-lobar resection, $17.2 \%$ SABR and $29.6 \%$ radical radiotherapy - differences not significant on uni-variable regression, which may relate to small patient numbers. Figure 1 demonstrates the sites of recurrence. Considering only those with pathological confirmation of cancer, recurrence rates at two years were $17.9 \%$ for SABR and $32.4 \%$ for radical radiotherapy.

Conclusions The lowest recurrence rate was observed following surgical resection. In comparison, recurrence following SABR was non-significantly higher due to more loco-regional recurrence. Radical radiotherapy is associated with higher rates of overall, loco-regional and distant recurrence. Nodal recurrence was comparable between lobectomy, SABR and radical radiotherapy. This data is limited by low numbers as well as the confounding effects of early non-cancer deaths and incomplete pathological confirmation in the non-surgical treatment cohorts.

\section{S65 DEVELOPING A MULTI-DISCIPLINARY THORACIC SURGERY RESEARCH TEAM IMPROVES THE RECRUITMENT INTO AND QUALITY OF CLINICAL TRIALS}

A Kerr, N Oswald, S Kadiri, H Bancroft, E Virgilio, J Webb, M Bellamy, J Taylor, E Bishay, M Kalkat, R Steyn, P Rajesh, B Naidu. Heart of England NHS Foundation Trust, Birmingham, UK

\subsection{6/thoraxjnl-2016-209333.71}

Objectives Recruitment into surgical trials historically has been fraught with difficulty. We examine whether developing a multidisciplinary research team has aided recruitment, data collection, patient retention and so success of clinical trials. In addition we look at effects on the patient experience of the surgical pathway. Methods We evaluated the development and impact of a specialist thoracic trained research team of nurses and allied health care professionals in a regional thoracic unit from 2009-2015. We assessed the impact on the recruitment into National Institute for Health Research Clinical Research Network (NIHR CRN) thoracic surgery portfolio trials. Patient experience was captured through a survey $(\mathrm{n}=30)$ and research team feedback through interviews $(\mathrm{n}=5)$.

During the development, clear leadership and support networks were created, new members were trained by specialist thoracic research nurses to obtain competences in both research and thoracic surgery to enable confident valid informed consent and the collection of robust quality data.

Results Since the development of a specialised thoracic surgery research team in 2010 the number trials have steadily increased and along with number of team members whilst clinical activity remained constant. The number of patient consented into clinical trials increased 7 fold (Table 1). From staff interviews a recurring theme was that a clear team structure and a specialist training aided them to be better patient advocate not only in research but in the clinical pathway. Patients universally agreed that involvement of the research team helped reduce their anxiety about their surgery and so enhanced patients experience.

Conclusion The impact of a dedicated research team goes well beyond research but improves clinical care. Having a clear support system and a specialist trained team has increased recruitment and retention into thoracic surgical trials and enhanced the patient's experience of research and the surgical pathway.

\begin{tabular}{|c|c|c|c|}
\hline $\begin{array}{c}\text { Abstract S65 Table 1 } \\
\text { Year } \\
\text { (April to April) }\end{array}$ & $\begin{array}{c}\text { CLRN Thoracic } \\
\text { Research Trials } \\
\text { open }\end{array}$ & $\begin{array}{c}\text { Number of } \\
\text { Thoracic research } \\
\text { staff }\end{array}$ & $\begin{array}{c}\text { Number of } \\
\text { consents in } \\
\text { thoracic surgery } \\
\text { research }\end{array}$ \\
\hline 2009 & 2 & 2 & 95 \\
\hline 2010 & 4 & 3 & 216 \\
\hline 2011 & 4 & 3 & 353 \\
\hline 2012 & 3 & 4 & 373 \\
\hline 2013 & 6 & 5 & 919 \\
\hline 2014 & 5 & 6 & 681 \\
\hline 2015 & 5 & 7 & 948 \\
\hline
\end{tabular}

\section{Improving Outcomes During COPD Hospitalisations}

\section{S66 LEVELS OF SALIVARY C-REACTIVE PROTEIN, PROCALCITONIN AND NEUTROPHIL ELASTASE CAN PREDICT EXACERBATIONS IN COPD AND DETERMINE THOSE PATIENTS AT HIGH RISK OF RE-EXACERBATION}

${ }^{1} \mathrm{~N}$ Patel, ${ }^{1} \mathrm{G}$ Thorpe, ${ }^{1} \mathrm{P}$ Jones, ${ }^{1} \mathrm{~V}$ Adamson, ${ }^{2} \mathrm{~J}$ Belcher, ${ }^{1} \mathrm{MA}$ Spiteri. ${ }^{1}$ Directorate of Respiratory Medicine, University Hospital of North Midlands, Stoke-on-Trent, UK; ${ }^{2}$ School of Computing and Mathematics, Keele University, Stoke-on-Trent, UK

\subsection{6/thoraxjnl-2016-209333.72}

Saliva has numerous practical advantages as a diagnostic bio-sample for management of long term conditions. We have previously demonstrated that C-reactive protein (CRP), procalcitonin (PCT) and neutrophil elastase (NE) can be reliably and reproducibly detected in saliva, offering useful information on health status. This study explores whether proactive monitoring of target analytes provides early warning of COPD exacerbations and re-exacerbation events.

Salivary CRP, PCT and NE levels were determined weekly in 55 subjects with established COPD, known to be frequent exacerbators [GOLD Stage I, 7; Stage II, 24; Stage III, 19; Stage IV, 5]. Daily symptom scores were collected using a self-assessment electronic diary. Participants were monitored throughout their stable, prodromal (defined as the 7 days prior to exacerbation onset), exacerbation and post-treatment recovery periods. All three salivary biomarkers could distinguish stable status from onset of patient-defined exacerbations (CRP: $\mathrm{p}<0.003$, PCT: $\mathrm{p}<0.001$, $\mathrm{NE}: \mathrm{p}<0.01$ ); CRP median increase was $1.82 \mathrm{ng} / \mathrm{ml}$, [interquartile range 4.79]; PCT $0.03 \mathrm{ng} / \mathrm{ml}$ [0.10] and NE $364 \mathrm{ng} / \mathrm{ml}$ [76]. Interestingly, increases over stable baseline were also observed in the prodromal period for salivary CRP $0.53 \mathrm{ng} / \mathrm{ml}$ [2.73], ( $\mathrm{p}<0.001)$, PCT $0.08 \mathrm{ng} / \mathrm{ml}$ [0.01], (p < 0.01) and NE $519 \mathrm{ng} /$ $\mathrm{ml}$ [568], ( $\mathrm{p}<0.007)$, occurring at $5.4 \pm 1.8$ days prior to patient-defined onset. Importantly, in those COPD patients experiencing more than 1 exacerbation $(\mathrm{n}=15)$, re-exacerbator salivary CRP was significantly higher at the index exacerbation, 4.08 $\mathrm{ng} / \mathrm{ml}[0.21]$, $(\mathrm{p}<0.04)$ with a mean time of $11 \pm 8$ days to reexacerbation after treatment completion. Diary symptom analysis 
showed subjective deterioration in symptom trajectory 4 days prior to exacerbation onset $(\mathrm{p}<0.01$ ), with re-exacerbators demonstrating a higher baseline symptom burden in the posttreatment period compared to single exacerbators $(p<0.01)$.

In conclusion, salivary biomarker levels can complement patient self-assessment to provide clinically useful cues to enable earlier identification of exacerbations in COPD; salivary CRP potentially offers additional information on re-exacerbation risk. These results support opportunities for patient-reported events and salivary biomarkers to be used synergistically in future nearpatient COPD diagnostics for enhanced self-management and prompt exacerbation intervention.

\section{\begin{tabular}{|l|l}
\hline S67 MORTALITY IN COPD PATIENTS FOLLOWING \\
\hline
\end{tabular} COMMUNITY ACQUIRED PNEUMONIA: A POPULATION DATABASE ANALYSIS OF LINKED HEALTHCARE RECORDS}

${ }^{1} \mathrm{~N}$ Williams, ${ }^{2} \mathrm{NA}$ Coombs, ${ }^{3} \mathrm{M}$ Johnson, ${ }^{2} \mathrm{~L}$ Josephs, ${ }^{4} \mathrm{LA}$ Rigge, ${ }^{2} \mathrm{DM}$ Thomas, ${ }^{5}$ TMA Wilkinson. 'Southampton NIHR Biomedical Research Unit, University Hospital Southampton NHS Foundation Trust, Southampton, UK; ${ }^{2}$ Primary Care and Population Sciences, University of Southampton, Southampton, UK; ${ }^{3}$ NIHR CLAHRC Wessex Methodological Hub, University of Southampton, Southampton, UK; ${ }^{4}$ Department of Respiratory Medicine, University Hospital Southampton NHS Foundation Trust, Southampton, UK; ${ }^{5}$ Clinical \& Experimental Sciences, University of Southampton, Southampton, UK

\subsection{6/thoraxjnl-2016-209333.73}

Introduction Community acquired pneumonia (CAP) is a common occurrence in patients with chronic obstructive pulmonary disease (COPD), yet controversy still remains about its affect on outcome. We therefore investigated the impact of CAP on mortality in a cohort of COPD patients identified from the Hampshire Health Record analytical database, a local NHS database containing linked, anonymised primary and secondary care records.

Methods Patients were defined as having COPD if they had a diagnostic Read code in their primary care record at any time prior to the $1^{\text {st }}$ January 2010 and were aged $\geq 40$ years at the start of the study. CAP episodes occurring over a 5 -year period from $1^{\text {st }}$ January 2010 were identified using Read and ICD-10 codes. The outcome measure was all-cause mortality following a CAP diagnosis. Cox proportional hazard modelling was used to estimate hazard ratios (HR) and confidence intervals (CI), adjusting for age, sex, GOLD stage, smoking status and inhaled corticosteroid use (ICS)).

Results The cohort comprised 14506 COPD patients. The mean age was $70.3( \pm 10.8)$ years and $53.6 \%$ were male. $1931(13.3 \%)$ patients had at least one CAP and 2870 (19.8\%) deaths occurred over the study period. $28.2 \%$ of patients diagnosed with CAP died compared to only $9.7 \%$ of those without a CAP diagnosis $(\mathrm{p}<0.001)$. Logistic regression analysis, controlling for potential confounders identified CAP as an independent risk factor for future mortality (odds ratio 2.72; CI 2.37-3.12, p < 0.001). Compared to younger individuals (40-59 years) those aged 6079 and $>80$ years had the highest mortality risk following CAP (HR 2.65; CI 1.61-4.34, HR 7.03; CI 4.27-11.57 respectively, both $\mathrm{p}<0.001)$. Concurrent use of inhaled Fluticasone or Budesonide were associated with reduced mortality risk following CAP (HR 0.82; CI: 0.68-0.98 p = 0.029, HR 0.55; CI: 0.39$0.76 \mathrm{p}<0.001$, respectively) (Figure 1).

Conclusion CAP in COPD is associated with increased risk of mortality, especially in older individuals. Although known to increase CAP risk, ICS use appears to reduce risk of mortality following CAP. Further research to understand the mechanisms underlying CAP risk in COPD and modulating effects of ICS is key, to guide development of future, targeted preventative strategies.

\section{S68 COPD IN THE ED: EOSINOPHILS, TREATMENT AND OUTCOMES, DATA FROM THE PRE-AWARD STUDY}

${ }^{1}$ REK Russell, ${ }^{2} \mathrm{~T}$ Doggett, ${ }^{1} \mathrm{I}$ Pavord, ${ }^{2} \mathrm{R}$ Pullinger, ${ }^{2} \mathrm{~S}$ Beer, ${ }^{1} \mathrm{M}$ Bafadhel. ${ }^{1}$ University of Oxford, Oxford, UK; ${ }^{2}$ Oxford University Hospitals NHS Foundation Trust, Oxford, UK

\subsection{6/thoraxjnl-2016-209333.74}

Rationale Acute exacerbations of COPD (AECOPD) are common. Peripheral blood eosinophil count (PBE) predicts outcomes in moderate and severe exacerbations, but little is known about PBE levels and outcomes of AECOPD in the emergency department (ED).

Methods Data for all attendances to ED of a large teaching hospital throughout a 12-month period for patients attending with AECOPD were studied. Anonymised data was cleaned to remove diagnostic and data errors and analysed using GraphPadPrism6 with statistical methods suitable for the data collected. Data is
A



B

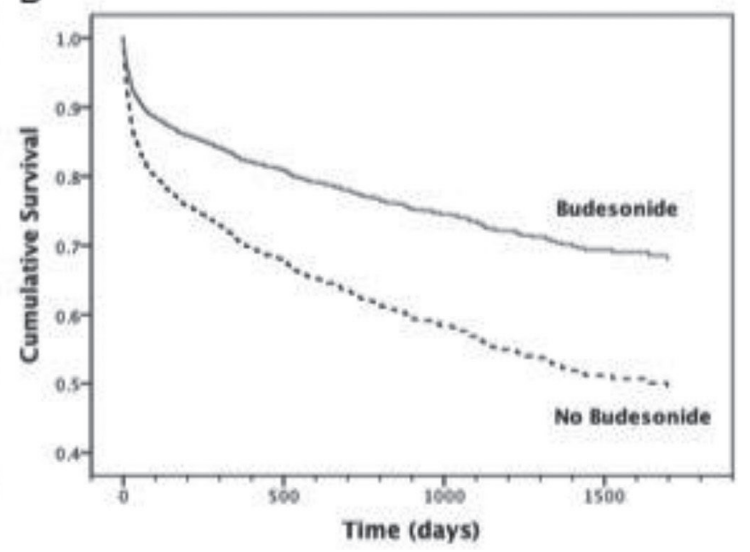

Abstract S67 Figure 1 Survival comparisons following CAP in COPD patients, stratified by Fluticasone (A) or Budesonide (B) use 